Pathologe $2021 \cdot 42: 305-309$

https://doi.org/10.1007/s00292-021-00936-7

Angenommen: 10. März 2021

Online publiziert: 14. April 2021

๑) Springer Medizin Verlag $\mathrm{GmbH}$, ein Teil von

Springer Nature 2021

Schwerpunktherausgeberin

E. Wardelmann, Münster

\author{
Amelie Friederike Büttcher ${ }^{1}$ Philipp Ströbel ${ }^{2}$ \\ 'Studiendekanat, Bereich Medizindidaktik, Universitätsmedizin Göttingen, Göttingen, Deutschland \\ ${ }^{2}$ Institut für Pathologie, Universitätsmedizin Göttingen, Göttingen, Deutschland
}

\title{
Digitale Lehre im Fach Pathologie
}

Multimedialität. Möglichkeit des Einsatzes unterschiedlicher Medien wie Bücher, elektronische Skripte, Audio- oder Videodateien etc. für die Wissensvermittlung.

Multimodalität. Möglichkeit des Nutzens unterschiedlicher Sinneswahrnehmungen („Kanäle“), zumeist auditive oder visuelle Eindrücke.

E-Learning ist über sehr unterschiedliche Formate bzw. Tools und in unterschiedlichen didaktischen Szenarien einsetzbar. Beispiele für Formate, Systeme bzw. Tools sind Lernsoftwares (z.B. digitale Anatomieatlanten), Simulationen, Lernspiele sowie Lernmanagementsysteme („learning management system“, LMS), auf denen der gesamte Prozess von Lehrveranstaltungen (Registrierung, Ablage von Lehrmaterialien, Erfolgskontrolle etc.) abgebildet werden kann. Je nach Unterrichtsszenario unterscheidet man die rein virtuelle Lehre mit online abrufbaren digitalen Inhalten (z.B. Lehrskripte, Vorlesungsfolien, Audiodateien/Podcasts) vom Blendedlearning-Format, bei denen virtueller Unterricht mit Präsenzlehre adäquat kombiniert wird [4]. Ein Beispiel für ein Blended-learning-Format ist das sog. Flipped-classroom-Modell, welches im Hochschulkontext Inverted-classroomModell genannt wird. Hierbei bereiten sich die Studierenden in einer vorgeschalteten Selbstlernphase auf eine sich anschließende Präsenzphase vor, in der das Wissen gemeinsam mit den Dozent*innen angewandt und vertieft wird [12]. Weitere Beispiele sind das Onlinelernen im Anschluss an Präsenzveranstaltungen oder digitale Arbeitsein- heiten wie Simulationen oder virtuelle Arbeitsgruppen während Präsenzveranstaltungen [4]. Die Vorteile solcher digitalen Formate sind vielfältig: Inhalte können multimedial und multimodal aufbereitet und dadurch anschaulicher vermittelt werden. Interaktive Elemente können den Anreiz, sich mit den Inhalten z.B. spielerisch zu beschäftigen, erhöhen und für die Lernerfolgskontrolle eingesetzt werden. Durch das orts- und zeitunabhängige Angebot kann der potenzielle Nutzerkreis deutlich erweitert (z.B. Berufstätige, Lernende mit Kindern etc.) und hierdurch das Lerntempo individualisiert angepasst werden. Nicht zuletzt erfordert das Wegfallen fester täglicher Termine ein erhebliches Maß an Selbstdisziplin und ggf. eine Anpassung der Arbeitsgewohnheiten. Didaktische Fehler bei der Gestaltung von digitalen Formaten oder ihr unkritischer Einsatz können außerdem den intendierten Lernerfolg erschweren oder ganz verhindern. Exemplarisch können hier die Verwendung einer monotonen Sprechweise, eine fehlende Lernzielkongruenz oder zeitlich zu lange Einheiten genannt werden. Bezüglich der idealen Länge von Lehrvideos existieren unterschiedliche Empfehlungen in der Literatur. Eine publizierte Übersichtsarbeit zum Inverted-classroom-Modell bezieht sich diesbezüglich auf die empfohlene Länge von Videos in MOOCs („massive open online courses") und empfiehlt eine Zeitspanne von 6 bis maximal $20 \mathrm{~min}$ pro Video [12].

Insbesondere vor dem Hintergrund der zunehmenden Digitalisierung in der Medizin können Studierende durch den Einsatz von E-Learning-Methoden be- 
reits während des Studiums wertvolle Kompetenzen im Umgang mit digitalen Ressourcen erlangen. Sie werden somit optimal auf ihre spätere berufliche Tätigkeit im digitalen Zeitalter vorbereitet $[6,7]$.

Ebenfalls muss berücksichtigt werden, dass bei der Anwendung von E-LearningMaßnahmen eine Reihe von technischen Voraussetzungen sowohl auf Seiten der Lehrenden als auch der Studierenden erfüllt sein müssen [4]. Hierzu zählen bspw. (mobile) Endgeräte, eine stabile Internetverbindung mit ausreichender Bandbreite sowie ggf. Equipment zur Aufnahme und/oderWiedergabe von Bild- und Toninhalten.

\section{Anwendungsbeispiele im Fach Pathologie im Medizinstudium}

Die aktuelle COVID-19-Pandemie hat dazu geführt, dass die Lehre in der Medizin modernisiert wurde und vielfach digitale Lehr- und Lernformate eingeführt wurden. Die Lehrenden wurden dazu animiert, sich mit E-Learning zu beschäftigen und neue multimediale Inhalte für ihre Lehre zu implementieren.

Auch die Lehre im Fach Pathologie bietet zahlreiche Möglichkeiten für den sinnvollen Einsatz von E-Learning-Methoden. Hierbei bietet sich bspw. der Inverted-classroom-Ansatz für die histologischen und makroskopischen Kurse an. Für die Planung bzw. die Etablierung eines entsprechenden Angebots empfiehlt sich die Orientierung am sog. Kern-Zyklus [1, 5]:

1. Problemidentifikation und globale Bedarfsanalyse,

2. spezifische Bedarfsanalyse der Zielgruppe,

3. Formulierung übergeordneter Ziele und spezifischer Lernziele,

4. Auswahl von Lehrmethoden,

5. Umsetzung,

6. Evaluation und Feedback.

Bezogen auf die Planung, z. B. eines hypothetischen einsemestrigen E-LearningCurriculums für den Bereich der allgemeinen Pathologie, könnte ein solcher Zyklus etwa wie folgt aussehen:
1. Problemidentifikation und globale Bedarfsanalyse. Aufgrund der COVID19-Pandemie wird am Universitätsstandort $\mathrm{N}$ die reguläre Präsenzlehre eingestellt und mit kurzer Vorlaufzeit die Umstellung des bisherigen Präsenzunterrichts aufE-Learning-Formate notwendig. Eine herkömmliche Lehre im Präsenzbetrieb mit den bisher eingesetzten analogen Lehrformaten ist nicht mehr möglich. Den Studierenden soll daraus kein Nachteil entstehen und ihnen die Lerninhalte trotz der Pandemie vermittelt werden. Dies ist mit den bisher eingesetzten Lehrformaten nicht möglich und betrifft auch die Lehre im Fach Pathologie.

Idealerweise sollte die Lehre daher über eine digitale und ortsunabhängige Wissensvermittlung erfolgen. Dieser Ansatz grenzt sich stark von der herkömmlichen Lehre im Präsenzbetrieb ab. Seine Implementierung stellt in Anbetracht der Kürze an verfügbarer Planungszeit und ggf. vorhandener Ressourcen für Lehrende und Lernende eine Herausforderung dar.

2. Bedarfsanalyse der Zielgruppe. Erfahrungsgemäß benötigen die Studierenden klar strukturierte Vorgaben bezüglich des Kursablaufs (organisatorische Aspekte) sowie des Erwartungshorizonts (inhaltliche Aspekte). Dies betrifft insbesondere Fristen sowie Kurs- und Prüfungsanforderungen. Wichtig ist, dass die Lernenden bereits zu Beginn des Kurses den Arbeitsaufwand abschätzen und entsprechend planen können.

Auf Seiten des Instituts und der Lehrenden besteht der Wunsch, die Studierenden umfassend auf ihre spätere Tätigkeit als Ärzt*innen vorzubereiten, eine angenehme Lern- und Lehratmosphäre $\mathrm{zu}$ schaffen, Wissensinhalte erfolgreich zu vermitteln und den jeweils standortspezifischen rechtlichen Vorgaben (bspw. Studienordnung) zu genügen. Jedoch muss mit ggf. bestehenden Vorbehalten gegenüber dem Einsatz von E-Learning-Methoden bei einzelnen Dozierenden gerechnet werden.

Um den Bedürfnissen der genannten Stakeholder gerecht zu werden, bietet es sich an, auf evtl. vorhandene digitale Ressourcen zurückzugreifen. Hierzu könnte bspw. ein am jeweiligen Standort bereits erfolgreich etabliertes LMS zählen, mit dem sich Onlinekurse erstellen und administrieren lassen (Ablage von Lehrmaterialien, Registrierung der Nutzer*innen, E-Prüfungen etc.). Unter Umständen besteht auch die Möglichkeit, auf digitale histologische Kurspräparate aus früheren Semestern oder andere Onlinematerialien des jeweiligen Instituts für Pathologie zurückzugreifen.

3. Formulierung übergeordneter Ziele und spezifischer Lernziele. Die Dozent $^{*}$ innen definieren unter Berücksichtigung des Nationalen Kompetenzbasierten Lernzielkatalogs/Kompetenzorientierten Gegenstandskatalogs (NKLM/GK) für den Kurs z. B. die folgenden übergeordneten und spezifischen Lernziele:

I. (übergeordnet): Die Absolvent ${ }^{*}$ innen nutzen Prinzipien der Pathogenese und Pathomechanismen zum Verständnis und zur Erklärung von Erkrankungen bzw. Symptomen sowie zur Ableitung von Therapieansätzen. Diesem übergeordneten Ziel sind weitere spezifische und messbare (z. B. in Klausuren) Lernziele unterzuordnen.

II. Spezifische Lernziele zu diesem Thema: Die Absolvent ${ }^{*}$ innen sollen am Beispiel der Pneumonie den Zusammenhang zwischen Virulenz des Erregers und Immunsituation des Wirts erklären können. Anhand makroskopischer und histologischer Präparate sollen die Studierenden die radiologischen, klinischen und pathohistologischen Unterschiede zwischen alveolären und interstitiellen Pneumonien erkennen und erläutern können. Basierend darauf sollen sie mögliche Komplikationen (z. B. Abszess, Sepsis, organisierende Pneumonie, Lungenfibrose) ableiten und daraus Grundzüge von therapeutischen Handlungsanweisungen konstruieren können (Wann ist der Einsatz von Antibiotika sinnvoll? Profitieren alle Patient ${ }^{*}$ innen mit Pneumonien von Sauerstoffgabe über die Atemwege? etc.). 
Die spezifischen Lernziele sollten in einem institutsinternen Lernziel- und Gegenstandskatalog festgehalten werden, der den Studierenden zusammen mit einem genauen Ablauf- und Stundenplan zu Beginn des Semesters kommuniziert wird (siehe Bedarfsanalyse).

4. Auswahl von Lehrmethoden. Es müssen geeignete Lehrmethoden unter Berücksichtigung der definierten Lernziele ausgewählt werden [8]. Die Dozierenden besprechen sich im Vorfeld mit den Administrator*innen des universitären LMS, um sich über die technischen Möglichkeiten der Lernplattform $\mathrm{zu}$ informieren und um den Bedarf an technischem Support festzustellen. Hierbei kann auf ggf. vorhandene Erfahrungen aus den vorangegangenen Semestern zurückgegriffen werden. Aus vorherigen Semestern könnte bspw. bekannt sein, dass vor allem der Umgang mit digitalen Slides ohne direkte Anleitung vielen Studierenden Probleme bereiten kann, was sich negativ auf die Lernmotivation und das Interesse am Fach auswirken kann. Insgesamt müssen auch die jeweiligen rechtlichen Vorgaben eingehalten werden.

Das Team beschließt, den Unterricht in thematisch aufeinander aufbauenden Einheiten zu organisieren, die aus einer Hauptvorlesung, thematisch flankiert von histologischen und makroskopischen Unterrichtseinheiten, bestehen soll. Unter der aktuellen Pandemiesituation und aufgrund des Social Distancing bietet es sich an, für dieses Vorhaben auf E-Learning-Szenarien zurückzugreifen. Die Hauptvorlesung ließe sich in Form von asynchronen Slidecasts (vertonte PowerPoint-Präsentationen) abbilden, die zusätzlich von einem (digitalen) Skript begleitet werden kann. Hierdurch können die Lernenden ortsunabhängig und zeitversetzt lernen, was ihnen mehr Flexibilität und Freiraum für individuelle Lerngewohnheiten einräumt. Aufgrund des eher konventionellen, „frontalen“ Ansatzes mit geringer Interaktionsmöglichkeit sollten zusätzliche Möglichkeiten des regelmäßigen Austausches über den vergangenen Lernstoff und der gezielten Vertiefung geschaffen werden. Hierbei sollte den Studierenden auch

Pathologe 2021 · 42:305-309 https://doi.org/10.1007/s00292-021-00936-7

(c) Springer Medizin Verlag GmbH, ein Teil von Springer Nature 2021

\section{A. F. Büttcher · P. Ströbel \\ Digitale Lehre im Fach Pathologie}

\section{Zusammenfassung}

Digitale Formate und Inhalte (E-Learning) sind längst fester Bestandteil der akademischen Lehre und der ärztlichen Weiterbildung geworden. Dies gilt auch für das Fach Pathologie. Digitale Formate bieten die Chance, den herkömmlichen Unterricht und auch Prüfungen durch zahlreiche neue Möglichkeiten abwechslungsreicher und anschaulicher zu gestalten und, z. B. durch die Unabhängigkeit von Ort und Zeit, den individuellen Bedürfnissen der Lernenden besser anzupassen. Zugleich gilt aber auch für diese Formate, dass sie sorgfältig geplant und in ein Lehrkonzept unter Berücksichtigung übergeordneter Kompetenzen und spezifischer Lernziele eingeordnet werden müssen und dass die technische Umsetzung geeignet sein muss, den didaktischen Zweck zu erfüllen. Nach aktueller Einschätzung werden (und sollten) virtuelle Angebote auch in Zukunft die Präsenzlehre nicht ersetzen, sondern sich gegenseitig in sog. Blendedlearning-Formaten ergänzen und bereichern. Es ist wichtig, den Einsatz solcher Methoden als lebendigen Prozess zu begreifen, der möglichst lerndidaktisch begleitet und durch regelmäßige Evaluationsmaßnahmen der Lehrenden und Lernenden weiterentwickelt werden sollte.

Schlüsselwörter

Computerunterstützter Unterrricht .

Fernlehre · Feedback · Medizinstudenten . Universitäten

\section{E-Learning in pathology}

\section{Abstract}

Digital formats have become an indispensable part of academic teaching, including education and training in pathology. Their use offers the perspective of rendering conventional teaching formats more diversified and more flexible since students can adopt the timing and learning speed to their individual needs. However, digital formats should be designed to fulfill the intended didactic purpose within a teaching concept that addresses competences and specific aims. The current view is that E-learning neither will nor should replace face-to-face teaching, but that both can fuse into blended-learning formats using the best of both worlds. It is important to view the implementation of E-learning as a dynamic process that should be underpinned by didactic research and be constantly developed further through evaluation and feedback from both teachers and students.

\section{Keywords}

Computer-assisted instruction · Distance education - Feedback - Medical students . Universities
Gelegenheit gegeben werden, Fragen $\mathrm{zu}$ stellen. Für die histologischen und makroskopischen Kurse sollten in einem Onlinemodul ggf. vorhandene oder noch zu erstellende digitale Präparate genutzt werden. Aufgrund möglicherweise bekannter Schwierigkeiten der Studierenden im Umgang mit den komplexen Präparaten bietet sich für die Lernvertiefung ein Inverted-classroomFormat an (- Abb. 1).

5. Umsetzung. Trotz der Pandemiesituation ist es wichtig, den Kontakt zu den Studierenden zu halten und den individuellen Lernfortschritt zu begleiten [9]. Hierfür müssen evtl. neue und auf die Erreichung der Lernziele ausgerichtete Lehrmaterialien erstellt bzw. bereitgestellt werden: Für die Hauptvorlesung werden Slidecasts genutzt, indem die Lehrenden kommentierte PowerPoint-Präsentationen aufzeichnen. Zentrale Inhalte des Unterrichts können darüber hinaus in einem ergänzenden Lernskript pointiert dargestellt werden. Für den Inverted-classroom-Ansatz der begleitenden histologischen und makroskopischen Kurse muss vorbereitendes Material, bspw. kurze Einleitungsvideos, in denen die Präparate vorgestellt und erläutert werden, erstellt werden. Zur Vervollständigung dieses Ansatzes können die Videos zusätzlich weiter- 


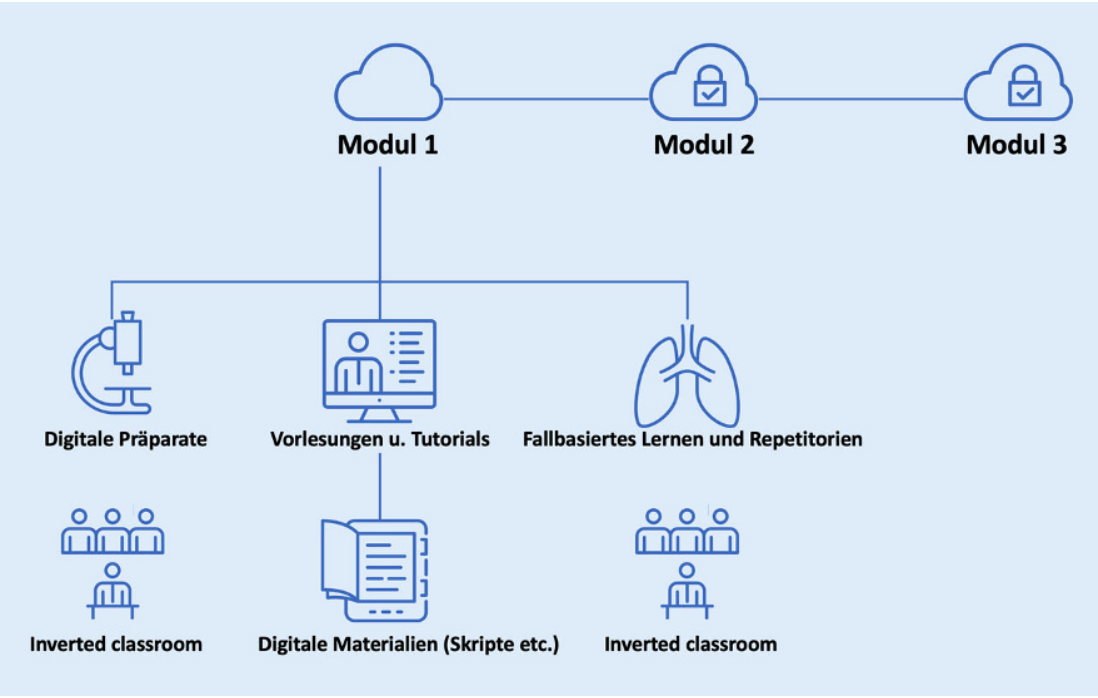

Abb. $1 \Delta$ Anwendungsbeispiel für ein Blended-learning-Curriculum mit der Kombination von digitalen Einheiten und Präsenzlehre im Fach Pathologie unter Verwendung unterschiedlicher Lernszenarien (klassischer Unterricht vs. Inverted Classroom) und unterschiedlicher Materialien (z. B. Skripte, Audiodateien und Lehrvideos, digitale Kurspräparate)

führende Fragen beinhalten, welche die Studierenden in Einzel- oder Gruppenarbeit bis zum digitalen Präsenztermin bearbeiten und individuelles Feedback darauf erhalten. Beim digitalen Präsenztermin erfolgt dann die Vertiefung der Lerninhalte. Durch die Nutzung standortübergreifender Synergien (siehe LOOOP-Projekt) ließe sich der Arbeitsaufwand für die einzelnen Institute ressourcenoptimiert gestalten ( $\bullet$ Abb.2).

6. Evaluation und Feedback. Am Ende des Kurses erfolgt die Überprüfung des individuellen Leistungsstands in Form einer Prüfung. Darüber hinaus sollte die Umsetzung der einzelnen Lehrmethoden des Kurses ebenfalls evaluiert und Feedback eingeholt werden. Hierfür sollte den Lernenden bereits während des Kurses ausreichend Gelegenheit gegeben werden, Probleme und Wünsche mit ihren Lehrenden zu besprechen und Kritik anzubringen. Darüber hinaus sollte eine schriftliche Evaluation am Ende des Kurses durchgeführt werden.

Für die Planung nachfolgender Semester sollte das Feedback der Lernenden strukturiert ausgewertet und berücksichtigt werden.

\section{Überlegungen zur Anwendung von E-Learning bei der Weiterbildung im Fach Pathologie}

Viele der oben erwähnten Grundsätze und Erfahrungen beim Einsatz von E-Learning gelten auch für die ärztliche Weiterbildung von Graduierten. Die verstärkte familiäre und berufliche Einbindung der Nutzer*innen und die beschränkte zeitliche Verfügbarkeit von qualifizierten Dozent*innen macht den Einsatz digitaler Medien besonders wünschenswert. Dem steht der Wunsch nach persönlichem Austausch in einer „geschützten“ Umgebung gegenüber, wie ihn bislang nur eine reelle Tagung bieten kann. Die Internationale Akademie für Pathologie (IAP) bietet beispielsweise schon seit Langem digitale Lehrsammlungen an, die dezentral bearbeitet und im Rahmen von Präsenzkursen vertieft werden können (https://www.iapbonn.de). Diese Angebote finden breiten Anklang und werden intensiv genutzt. Es wäre denkbar, solche Angebote im Austausch mit den nationalen und internationalen Fachgesellschaften zu standardisieren und zu einer zertifizierten curricularen digitalen Weiterbildung auszubauen.

Eine weitere interessante Perspektive wäre es, Fallserien, die von Expert ${ }^{\star}$ innen in bestimmten Fachgebieten über Jahrzehnte aufgebaut wurden, zu digitalisieren und zentral zu kuratieren und damit Spezialwissen für nachfolgende Generationen $\mathrm{zu}$ erhalten. Es könnte sich für die Fachgesellschaften lohnen, über den Aufbau von kooperativen Strukturen nachzudenken, mit denen dieses ambitionierte und sehr aufwendige Vorhaben (vermutlich mit externer Förderung) in die Tat umgesetzt werden können.

Überhaupt bieten die Möglichkeiten für eine standortübergreifende Vernetzung (z.B. im Rahmen der LOOOPPlattform [Learning Opportunities, Objectives and Outcomes Platform, https://looop.charite.de]) weitreichende neue Ansätze für die Ausbildung. Durch die professionelle und auf Ergebnissen aus der Lehrforschung basierende Weiterentwicklung von E-Learning-Materialien sowie zentrale Zusammenführung von Lehrmaterialien zu einem „Werkzeugkasten" wird es auch Standorten mit wenig entwickelter E-Learning-Kultur ermöglicht, eine inhaltlich hochwertige digitale Lehre durchzuführen, gleichzeitig bleibt den Standorten die Möglichkeit der individuellen Gestaltung ihrer Lehre unbenommen (• Abb. 2).

Zur Erhöhung der Interaktivität bei synchronen Veranstaltungen online sowie in Präsenz bietet es sich an, die Lernenden unter Anwendung von digitalen Tools aktiv einzubinden. Hierfür können bspw. sogenannte Votingtools, eine digitale Chatwall oder Quizsysteme zur Aktivierung eingesetzt werden.

Weitere hilfreiche Informationen und konkrete Tipps für praktisch interessierte Leser*innen bietet darüber hinaus die Website des Hochschulforums Digitalisierung (https:// hochschulforumdigitalisierung.de/).

\section{Fazit für die Praxis}

- E-Learning-Methoden gerade im Fach Pathologie dazu beigetragen, eine qualitativ hochwertige Lehre auch unter den Bedingungen der COVID19-Pandemie zu ermöglichen.

- Typischen Vorteilen des digitalen Lernens (z. B. orts- und zeitunabhängiger Zugriff auf die Inhalte) stehen spezifische Nachteile (tech- 


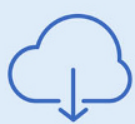

Skripte und andere Lehrmaterialien zum Herunterladen

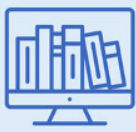

Online-Kurse mit verlinkten Inhalte

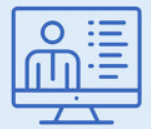

Videos, Web-Tutorials Audidateien etc.

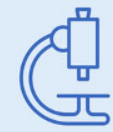

Digitale Slides und virtuelle makroskopische Präparate

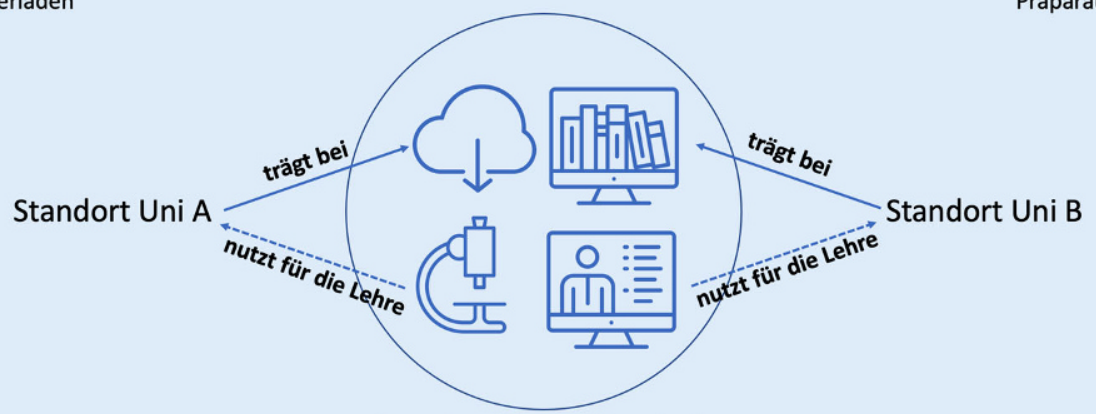

Standort Uni C (Hosting)

Abb. $2 \triangleleft$ Beispiele für sinnvolle digitale Materialien im Fach Pathologie und für die gemeinsame standortübergreifende Nutzung durch Vernetzung nologische Barrieren, unpersönliche Lernumgebung etc.) gegenüber.

- E-Learning sollte herkömmlicher Lehr- und Lernprozesse nicht voll ersetzen. Daher kombinieren die meisten aktuellen Ansätze virtuelle Angebote mit Präsenzlehre zu sog. Blended-learning-Formaten.

- Planung und Durchführung von E-Learning-Unterricht erfordert eine sorgfältige Vorbereitung und sollte sich z. B. am sog. Kern-Zyklus orientieren.

- E-Learning-Einheiten sollten dort eingesetzt werden, wo sie didaktisch sinnvoll sind. Auch digitale Inhalte müssen sich an allgemeingültigen didaktischen Grundkonzepten wie dem der Verständlichkeit orientieren.

\section{Korrespondenzadresse}

Prof. Dr. Philipp Ströbel
Institut für Pathologie,
Universitätsmedizin
Göttingen
Robert-Koch-Str. 40,
37075 Göttingen,
Deutschland
philipp.stroebel@med.uni-
goettingen.de

\section{Einhaltung ethischer Richtlinien}

Interessenkonflikt. A. F. Büttcher undP. Ströbel geben an, dass kein Interessenkonflikt besteht.

Für diesen Beitrag wurden von den Autoren keine Studien an Menschen oder Tieren durchgeführt. Für die aufgeführten Studien gelten die jeweils dort angegebenen ethischen Richtlinien.

\section{Literatur}

1. Chen BY, Kern DE, Kearns RM et al (2019) From modules to MOOCs: application of the six-step approach to Online curriculum development for medical education. Acad Med 94:678-685

2. Grissom S, Mcnally MF, Naps T (2003) Algorithm visualization in CS education: comparing levels of student engagement. In: Proceedings of the 2003 ACM symposium on Software visualization, S87-94

3. Guiter GE, Sapia S, Wright Al et al (2021) Development of a remote online collaborative medical school pathology curriculum with clinical correlations, across several international sites, through the Covid-19 pandemic. Med Sci Educ. https://doi.org/10.1007/s40670-021-01212-2

4. Hege I, Tolks D, Adler M et al (2020) Blended learning: ten tips on how to implement it into a curriculum in healthcare education. GMS J Med Educ 37:Doc45

5. Kern DE, Thomas PA, Hughes MT (2009) Curriculum development for medical education: a six-step approach. Johns Hopkins University Press,

6. Kuhn S, Frankenhauser S, Tolks D (2018) Digital learning and teaching in medical education : already there or still at the beginning? Bundesgesundheitsblatt Gesundheitsforschung Gesundheitsschutz 61:201-209

7. Kuhn S, Jungmann F (2018) Medicine in the digital age : Telemedicine in medical school education. Radiologe 58:236-240

8. Langer I, Schulz Von Thun F, Tausch R (2015) Sich verständlich ausdrücken. Ernst-Reinhard Verlag, München, Basel
9. Regmi K, Jones $L$ (2020) A systematic review of the factors-enablers and barriers-affecting e-learning in health sciences education. BMC Med Educ 20:91

10. Rey GD (2009) E-Learning: Theorien, Gestaltungsempfehlungen und Forschung. Huber,

11. Tabatabai S (2020) COVID-19 impact and virtual medical education. J Adv Med Educ Prof 8:140-143

12. Tolks D, Schafer C, Raupach T et al (2016) An introduction to the inverted/flipped classroom model in education and advanced training in medicine and in the healthcare professions. GMS J Med Educ 33:Doc46 